\title{
INDICADORES AMBIENTAIS E FINANCEIROS DO RETROFIT DO SISTEMA DE AR CONDICIONADO DE UMA AGÊNCIA BANCÁRIA
}

\section{ENVIRONMENTAL AND FINANCIAL INDICATORS OF THE RETROFIT OF HVAC SYSTEM OF A BANK BRANCH}

\section{Marcio Ferreira de Lima ${ }^{1}$; Ana Paula Dalla Corte}

\author{
${ }^{1}$ Especialização em Projetos Sustentáveis, Mudanças Climáticas e Mercado de \\ Carbono na Universidade Federal do Paraná, Curitiba/PR, \\ marciolima750@gmail.com \\ ${ }^{2}$ Setor de Ciências Agrárias na Universidade Federal do Paraná, Curitiba/PR, \\ anapaulacorte@gmail.com
}

\begin{abstract}
RESUMO
O objetivo deste artigo é analisar alguns indicadores ambientais e financeiros da obra de retrofit do sistema de ar condicionado de uma agência bancária. O edifício está localizado no centro da cidade de Florianópolis, SC. A substituição do sistema ocorreu no ano de 2012. O método analisou os resultados do ano de 2013 em comparação com os anos anteriores quanto aos aspectos de: eficiência energética, mitigação de emissões de gases de efeito estufa (GEE) e análise financeira do investimento. Os resultados foram: economia de 24,18\% de energia elétrica e mitigação de 51,31 $\mathrm{tCO}_{2}$ eq das emissões de GEE que são equivalentes ao plantio de 536 árvores. A análise financeira demonstrou que o retrofit é vantajoso com índice benefício/custo de 0,60 e payback de 14,85 anos. Conclui-se que um ano após a execução da obra de retrofit foi atingido o objetivo esperado, que era substituir o sistema de ar condicionado antigo por um novo com mais eficiência energética, redução do consumo de energia elétrica e a consequente redução do impacto ambiental.

Palavras-chave: Eficiência energética. Engenharia econômica. Inventário de gases de efeito estufa. Mudanças climáticas. Sustentabilidade.

\footnotetext{
ABSTRACT

The purpose of this study was to analyze some environmental and financial indicators of the retrofit installation in the heating, ventilating and air conditioning system (HVAC) of a bank branch. The building is located in downtown Florianópolis, SC, Brazil. The replacement of the system occurred in 2012. The method analyzed the results of the year 2013 compared with previous years in the matters of: energy efficiency, mitigation of greenhouse gases (GHG) and financial investment analysis. The results were: $24.18 \%$ electricity savings and $51.31 \mathrm{tCO}_{2} \mathrm{eq}$ mitigation of GHG emissions that are equivalent to planting 536 trees. The financial analysis showed that the retrofit is advantageous with benefit-cost ratio 0.60 and 14.85 years payback period. It was concluded that one year after the implementation of the retrofit was achieved the expected objective, which was to replace the old HVAC system with a new one more energy efficient, reducing energy consumption and the resulting reduction of the environmental impact.
} 
Keywords: Energy efficiency in buildings. Economic engineering. GHG inventory. Climate changes. Sustainability.

\section{INTRODUÇÃO}

O $5^{\circ}$ relatório do Painel Intergovernamental sobre mudanças climáticas (Intergovernmental Panel on Climate Change - IPCC), no documento Sumário para Formuladores de Políticas impactos, adaptação e vulnerabilidade (Impacts, Adaptation and Vulnerability - Summary for Policymakers) cita que a mudança climática é projetada para reduzir a demanda de energia para aquecimento e aumento da demanda para refrigeração nos setores residencial e comercial. No último e mais agravante cenário, RCP 8.5, no qual as emissões continuam a crescer em ritmo acelerado, há previsão de um armazenamento adicional de $8,5 \mathrm{~W} / \mathrm{m}^{2}$. Em tal situação a superfície da Terra poderia aquecer entre $2,6^{\circ} \mathrm{C}$ e $4,8^{\circ} \mathrm{C}$ ao longo deste século, até 2100 (IPCC, 2014a).

A Política Nacional sobre Mudança do Clima oficializa o compromisso voluntário do Brasil, junto à Convenção-Quadro da ONU sobre Mudança do Clima, de redução de emissões de gases de efeito estufa entre 36,1\% e 38,9\% do projetado até 2020 (Lei $n^{\circ} 12.187$ de 2009). O Decreto $n^{\circ}$ 7.390 de 2010, que regulamenta a lei, cita várias ações que serão implementadas para alcançar a redução de emissões, das quais pode-se citar a eficiência energética.

Retrofits constituem uma peça-chave da estratégia de mitigação em países com estoques de edifícios. Casos individuais atingem reduções de 50 a $90 \%$ de energia para aquecimento/arrefecimento. Grandes melhorias recentes no desempenho e baixos custos tornam os edifícios de baixo consumo energético e retrofits economicamente atrativos, às vezes até com custos líquidos negativos (IPCC, 2014b).

O retrofit do sistema de ar condicionado consiste na substituição de equipamentos antigos por novos modelos. Devido à atualização tecnológica os novos equipamentos consomem menos energia elétrica para produzir a mesma capacidade frigorígena, ou seja, tem mais eficiência energética.

Os equipamentos de ar condicionado da agência bancária apresentavam falhas constantes causando desconforto aos clientes e aumento do custo com manutenção corretiva. A partir do controle do consumo de energia elétrica da agência bancária verificou-se o aumento do consumo em relação ao histórico de anos anteriores. Considerou-se a restrição ao uso do gás refrigerante do sistema antigo, o HCFC-22 pelo Protocolo de Montreal, por ser agressivo à camada de ozônio e a opção do novo sistema com gás comercialmente conhecido como "gás ecológico", o R410-A. Estes argumentos motivaram a execução do retrofit do sistema.

O objetivo deste artigo é analisar alguns aspectos da sustentabilidade da obra de retrofit do sistema de ar condicionado de uma agência bancária. A substituição do sistema ocorreu no ano de 2012. O método analisou os resultados do ano de 2013 em comparação com os anos anteriores quanto aos aspectos de: eficiência energética, mitigação de emissões de gases de efeito estufa (GEE) e análise financeira do investimento.

\section{MATERIAL E MÉTODOS}

Analisou-se o histórico de consumo de energia elétrica da agência bancária de dois anos anteriores e de um ano posterior ao retrofit do sistema de ar condicionado para quantificar a eficiência energética e a redução de custos. Para a elaboração do inventário de emissões de GEE utilizou-se a metodologia do GHG Protocol Brasil (2012), dados de referência do IPCC (2014) e inventários de emissões de obras de construção civil (SANQUETTA et al., 2013). 


\subsection{Descrição da edificação}

Trata-se de um edifício comercial localizado no centro de Florianópolis (latitude $27^{\circ} 35^{\prime} \mathrm{S}$, longitude $48^{\circ} 32^{\prime} \mathrm{O}$ e altitude de $7 \mathrm{~m}$ acima do nível do mar), capital do estado de Santa Catarina. A agência bancária está instalada neste edifício desde o ano de 2001. Ocupa três pavimentos: térreo, primeiro pavimento e subsolo. A área total construída é de 1.603,85 $\mathrm{m}^{2}$ e área útil (descontadas as paredes e a estrutura) é de $1.593,00 \mathrm{~m}^{2}$.

O horário de funcionamento aberto ao público é de segunda a sexta-feira das $10 \mathrm{~h}$ às $16 \mathrm{~h}$, incluindo os trabalhos internos; o horário de trabalho é das $8 \mathrm{~h}$ às $18 \mathrm{~h}$. A sala de autoatendimento funciona sete dias por semana, das $8 \mathrm{~h}$ às $20 \mathrm{~h}$. A agência possui 71 postos de trabalho (mesas de atendimento ao público, guichês de caixa e estações de trabalho interno). No período de estudo dos anos de 2010 a 2013, não foram realizadas alterações na envoltória do imóvel, no sistema de iluminação ou equipamentos. A manutenção dos equipamentos de ar condicionado é realizada por empresa especializada com Plano de Manutenção, Operação e Controle (PMOC) que inclui ações corretivas e rotinas de manutenção preventiva e preditiva.

O sistema de ar condicionado da situação anterior ao retrofit era composto por 21 equipamentos. Uma central com quatro equipamentos tipo self contained. Os demais equipamentos eram do tipo mini-split, conforme definições da NBR 16.401-1, Instalações de ar-condicionado (ABNT, 2008). Os mini-splits são utilizados em ambientes que tem horário diferenciado, como a sala técnica da agência, que possui equipamentos de informática que funcionam 24 horas por dia, e a sala de autoatendimento, que funciona sete dias por semana, das $8 \mathrm{~h}$ às $20 \mathrm{~h}$. A capacidade total nominal de resfriamento é de $325,3 \mathrm{~kW}$ a carga de gás de $92,5 \mathrm{~kg}$. Os dados foram obtidos da placa de identificação dos equipamentos instalados. $O$ gás refrigerante utilizado era o hidroclorofluorcarboneto HCFC-22. Atualmente muito utilizado em instalações comerciais, mas com restrições ao uso pelo Protocolo de Montreal (agressivo à camada de ozônio), tem cronograma de eliminação iniciado pelo Ministério do Meio Ambiente (MMA, 2012) a partir de 2013 e eliminação progressiva até 2040. No ano de 2012 foi realizada a obra de retrofit do sistema. A obra foi iniciada no mês de abril e concluída em novembro.

O sistema do retrofit é do tipo multi-split VRV: Sistema central em que um conjunto de unidades de tratamento do ar de expansão direta, geralmente instaladas dentro do ambiente a que servem (designadas unidades internas), cada uma operada e controlada independentemente das demais, é suprido em fluído refrigerante líquido em vazão variável (VRV) por uma unidade condensadora central, instalada externamente (designada unidade externa) (ABNT, 2008). O sistema é composto por nove condensadoras (unidades externas) e 40 evaporadoras (unidades internas) tipo split-cassete. O gás refrigerante do novo sistema é o R-410A, um HFC (hidrofluorocarboneto) não agressivo à camada de ozônio (MMA, 2012). A capacidade total nominal de resfriamento é de $268,4 \mathrm{~kW}$ e a carga de gás é de $60,5 \mathrm{~kg}$. Os dados foram obtidos da placa de identificação dos equipamentos instalados.

\subsection{Consumo de energia elétrica}

$\mathrm{O}$ histórico de consumo de energia elétrica em quilowatts-hora (kWh) de dois anos anteriores, 2010 a 2011, no ano do retrofit, 2012 e um ano posterior, 2013, esta demonstrado na Tabela 1. Os dados foram obtidos das faturas de energia elétrica da concessionária local. O histórico do custo, em Reais (R\$), da energia elétrica dos anos de 2010 a 2013 está demonstrado na Tabela 2. 
Tabela 1 - Consumo mensal de energia elétrica (kWh).

\begin{tabular}{lllllllllllllll}
\hline \multirow{2}{*}{ Ano } & \multicolumn{10}{c}{} & \multicolumn{10}{c}{ Jan } & Fev & Mar & Abr & Mai & Jun & Jul & Ago & Set & Out & Nov & Dez & Total \\
\hline 2010 & 26.739 & 30.878 & 30.537 & 27.115 & 23.438 & 16.983 & 19.030 & 16.437 & 22.967 & 22.710 & 27.223 & 30.276 & $\mathbf{2 9 4 . 3 3 3}$ \\
\hline 2011 & 29.312 & 34.047 & 30.617 & 28.106 & 26.870 & 21.817 & 19.054 & 22.548 & 19.753 & 21.300 & 22.602 & 29.788 & $\mathbf{3 0 5 . 8 1 4}$ \\
\hline 2012 & 28.294 & 33.933 & 32.669 & 26.936 & 27.033 & 22.824 & 22.609 & 18.655 & 21.049 & 23.289 & 21.849 & 23.186 & $\mathbf{3 0 2 . 3 2 6}$ \\
\hline 2013 & 21.872 & 21.219 & 23.211 & 21.258 & 22.896 & 15.360 & 17.180 & 16.841 & 14.885 & 16.123 & 18.982 & 17.694 & $\mathbf{2 2 7 . 5 2 1}$ \\
\hline
\end{tabular}

Fonte: Faturas da concessionária.

Tabela 2 - Custo mensal de energia elétrica (R\$).

\begin{tabular}{|c|c|c|c|c|c|c|c|c|c|c|c|c|c|}
\hline \multirow{2}{*}{ Ano } & \multicolumn{12}{|c|}{ Mês } & \multirow{2}{*}{ Total } \\
\hline & Jan & Fev & Mar & Abr & Mai & Jun & Jul & Ago & Set & Out & Nov & Dez & \\
\hline 2010 & 9.031 & 10.690 & 10.224 & 9.074 & 8.503 & 6.489 & 7.349 & 6.586 & 9.710 & 9.753 & 11.201 & 11.623 & 33 \\
\hline 2011 & 0.279 & 1.981 & 11.383 & 10.923 & 10.779 & 9.156 & 8.429 & 9.705 & 8.902 & 9.282 & 9.027 & 10.784 & 330 \\
\hline 2012 & 11.043 & 13.174 & 12.595 & 10.970 & 11.473 & 9.521 & 9.753 & 8.453 & 9.247 & 10.5 & 9.1 & 9.377 & \\
\hline 2013 & 8.668 & 7.657 & 7.712 & 7.220 & 7.847 & 5.392 & 6.155 & 6.579 & 6.211 & 6.721 & 7.212 & 6.911 & 84.286 \\
\hline
\end{tabular}

Fonte: Faturas da concessionária.

\subsection{Inventário de emissões de GEE}

Para a elaboração do inventário utilizou-se a metodologia do GHG Protocol que é hoje a ferramenta mais utilizada mundialmente pelas empresas e governos para entender, quantificar e gerenciar suas emissões. O Programa Brasileiro GHG Protocol é uma iniciativa do Centro de Estudos em Sustentabilidade, da Fundação Getulio Vargas (FGV), e do World Resources Institute (WRI), em parceria com o Ministério do Meio Ambiente (MMA), o Conselho Empresarial Brasileiro para o Desenvolvimento Sustentável (CEBDS) e o World Business Council for Sustainable Development (WBCSD) (GHG, 2012). Os inventários são divididos em: Escopo 1, Escopo 2 e Escopo 3, conforme descrito a seguir:

O Escopo 1 contabiliza as emissões diretas de GEE da própria empresa ou instituição (emissões físicas). As emissões fugitivas incluem o vazamento de gás refrigerante durante o uso de equipamento de refrigeração e ar condicionado (GHG, 2012).

O Escopo 2 contabiliza as emissões de GEE provenientes da aquisição de energia elétrica e térmica que é consumida pela empresa. A energia adquirida é definida como sendo aquela que é comprada ou então trazida para dentro dos limites organizacionais da empresa. No Escopo 2 as emissões ocorrem fisicamente no local onde a energia é produzida, quando a produção ocorre fora do limite organizacional (GHG, 2012).

Os fatores de emissão para o consumo de energia elétrica foram baseados no Sistema Interligado Nacional (SIN), os valores são calculados e fornecidos pelo Ministério de Ciência e Tecnologia (MCT, 2013). Na Tabela 3 estão descritos os valores utilizados para o cálculo da energia elétrica.

Tabela 3 - Fator Médio Mensal ( $\left.\mathrm{tCO}_{2} / \mathrm{MWh}\right)$ do Sistema Interligado Nacional (SIN).

\begin{tabular}{|c|c|c|c|c|c|c|c|c|c|c|c|c|c|}
\hline \multirow{2}{*}{ Ano } & \multicolumn{12}{|c|}{ Mês } & \multirow{2}{*}{$\begin{array}{c}\text { Média } \\
\text { anual }\end{array}$} \\
\hline & Jan & Fev & Mar & Abr & Mai & Jun & Jul & Ago & Set & Out & Nov & Dez & \\
\hline 2013 & 0,1151 & 0,1090 & 0,0981 & 0,0959 & 0,1151 & 0,1079 & 0,0838 & 0,0833 & 0,0840 & 0,0831 & 0,0930 & 0,0841 & 0,0960 \\
\hline
\end{tabular}

Fonte: Ministério da Ciência e Tecnologia, 2013. 
Com a equação (1) calculam-se as emissões da energia elétrica consumida (SANQUETTA et al., 2013):

$$
\text { Emissões }=F e . E e
$$

Onde:

Emissões = emissão de $\left(\mathrm{tCO}_{2}\right)$;

$\mathrm{Fe}=$ fator de emissão de emissão nacional $\left(\mathrm{tCO}_{2} / \mathrm{MWh}\right)$;

$\mathrm{Ee}=$ energia elétrica $(\mathrm{MWh})$.

O Escopo 3 é uma categoria de relato opcional, que permite a consideração de todas as outras emissões indiretas. A contabilização das emissões de Escopo 3 não precisa envolver uma análise completa do ciclo de vida de GEE de todos os produtos e operações. Normalmente, é útil concentrar-se em uma ou duas das maiores atividades geradoras de GEE (GHG, 2012).

Neste trabalho foi considerada a fonte de emissões relativa ao descarte de produtos vendidos ao fim de sua vida útil, devido à quantidade significante de gás nos equipamentos da situação anterior ao retrofit.

\subsection{Indicadores ambientais}

O uso de indicadores em forma de razão (quocientes) fornece informações sobre desempenho relativamente a um tipo de atividade e podem facilitar comparações entre produtos e processos semelhantes ao longo do tempo. As organizações podem resolver relatar indicadores de GEE em forma de razão com a finalidade de: avaliar o desempenho ao longo do tempo; por exemplo, estabelecer relações entre valores de diferentes anos, identificar tendências nos dados e apresentar o desempenho em relação a objetivos e anos-base (GHG, 2012).

A iniciativa CarbonBuzz do Royal Institute of British Architects e o Chartered Institution of Building Services Engineers no Reino Unido relacionam o consumo de energia e as emissões de GEE em edifícios. O centro da distribuição dos dados é o fator de emissões de 69,2 $\mathrm{kgCO}_{2} \mathrm{eq} / \mathrm{m}^{2} / \mathrm{ano}$.

O fator de emissão de carbono da ferramenta CarbonBuzz relaciona as emissões de carbono equivalente anual $\left(\mathrm{kgCO}_{2} \mathrm{eq} / \mathrm{ano}\right)$ durante a operação da edificação por metro quadrado de área útil $\left(\mathrm{m}^{2}\right)$. Com a equação (2) calcula-se a emissão de carbono anual $\left(\mathrm{kgCO}_{2} \mathrm{eq} / \mathrm{m}^{2} / \mathrm{ano}\right)$.

$$
\mathrm{Fe}=\frac{\mathrm{CO}_{2}}{\mathrm{Au}}
$$

Onde:

$\mathrm{Fe}=$ Fator de emissão de carbono $\left(\mathrm{kgCO}_{2} \mathrm{eq} / \mathrm{m}^{2} / \mathrm{ano}\right)$;

$\mathrm{CO}_{2}=$ Emissão total de GEEs anual $\left(\mathrm{kgCO}_{2} \mathrm{eq} / \mathrm{ano}\right)$;

$\mathrm{Au}=$ Área útil da edificação $\left(\mathrm{m}^{2}\right)$.

Modelos de compensação de emissões por meio de plantios florestais foram propostos por Flizikowski (2012). A quantidade de espécies arbóreas é calculada pela equação (3), com a quantidade total de GEE emitido em função das atividades e o fator de fixação de carbono em biomassa. No modelo de reflorestamento com a espécie Eucalyptus grandis o fator de fixação obtido foi de 10,45 árvores/ $\mathrm{tCO}_{2}$ eq.

$$
N=E t . F f
$$

Onde:

$\mathrm{N}=$ Quantidade de árvores a serem plantadas;

$\mathrm{Et}=$ Emissão total de GEEs estimada $\left(\mathrm{tCO}_{2} \mathrm{eq}\right)$;

$\mathrm{Ff}=$ Fator de fixação de carbono em biomassa (árvores/ $/ \mathrm{tCO}_{2} \mathrm{eq}$ ). 
Borgstein e Lamberts (2014) utilizaram o indicador anual quilowatt-hora por metro quadrado de área útil ( $\left.\mathrm{kWh} / \mathrm{m}^{2} / \mathrm{ano}\right)$ demonstrado na equação (4).

$$
F c=\frac{C e}{A u}
$$

Onde:

$\mathrm{Fc}=$ Fator de consumo de energia elétrica $\left(\mathrm{kWh} / \mathrm{m}^{2} / \mathrm{ano}\right)$;

$\mathrm{Ce}=$ Consumo de energia elétrica anual $(\mathrm{kWh} / \mathrm{ano})$;

$\mathrm{Au}=$ Área útil da edificação $\left(\mathrm{m}^{2}\right)$.

Na plataforma de cálculo do benchmarking de agências bancárias publicada no site do Conselho Brasileiro de Construção Sustentável estão disponíveis os indicadores de consumo com correção climática de 413 municípios brasileiros. Para o clima do município de Florianópolis o valor médio é de 193,25 kWh/m²/ano (CBCS, 2014).

\subsection{Indicadores financeiros}

Adotou-se como investimento inicial a soma dos valores dos equipamentos, mão-de-obra, transportes, lucro da empresa contratada e impostos devidos. Com a equação (5) calcula-se o custo da energia elétrica consumida:

$$
\text { Custo }=T . E e
$$

Onde:

Custo $=$ reais $(\mathrm{R} \$)$;

$\mathrm{T}=$ custo unitário $(\mathrm{R} \$ / \mathrm{kWh})$;

$\mathrm{Ee}=$ consumo de energia elétrica $(\mathrm{kWh})$.

O Valor Presente Líquido (VPL) consiste em concentrar na data zero o valor presente de todos os fluxos de caixa do investimento descontados pela TMA (taxa mínima de atratividade), evidenciando o lucro econômico do projeto, conforme a equação (6) (CAMARGO, 2007).

$$
V P L=-C F_{0}+\sum \frac{C F_{j}}{(1+T M A)^{j}}
$$

Onde:

VPL $=$ Valor Presente Líquido

$\mathrm{CF}_{0}=$ Valor do investimento inicial

$\mathrm{CF}_{\mathrm{j}}=$ Cada Fluxo de Caixa nos ${ }_{\mathrm{j}}$ períodos

O Índice Benefício/Custo (IBC), também chamado de índice de lucratividade, mostra o valor que a empresa obtém para cada $\mathrm{R} \$ 1,00$ investido em um determinado projeto. Para tanto, devem ser relacionados os dispêndios de capital com os fluxos de benefícios resultantes de investimento, descontados pela TMA, conforme a equação (7) (CAMARGO, 2007).

$$
I B C=\frac{\text { Valor presente das entradas de caixa }}{\text { Valor presente das saídas de caixa }}
$$

O período de payback é o tempo de retorno de investimento, ou seja, o tempo que o investimento inicialmente despendido leva para ser recuperado. Para fluxos de caixa constantes, o 
cálculo pode ser simplificado, pois, para encontrar o payback, basta dividir o valor de investimento inicial pelo valor de entrada, conforme a equação (8) (CAMARGO, 2007).

$$
\text { payback }=\frac{\text { investimento inicial }}{\text { valor da entrada }}
$$

Foram consideradas duas situações alternativas:

- Anterior: Manter o sistema existente sem custo de reinvestimento.

- Retrofit: Equipamento novo com reinvestimento.

\section{RESULTADOS E DISCUSSÃO}

\subsection{Eficiência energética}

Na Figura 1, com base nos dados de consumo de mensal de energia elétrica, da Tabela 1, percebe-se a redução de consumo com destaque para o ano de 2013 (período posterior ao retrofit).

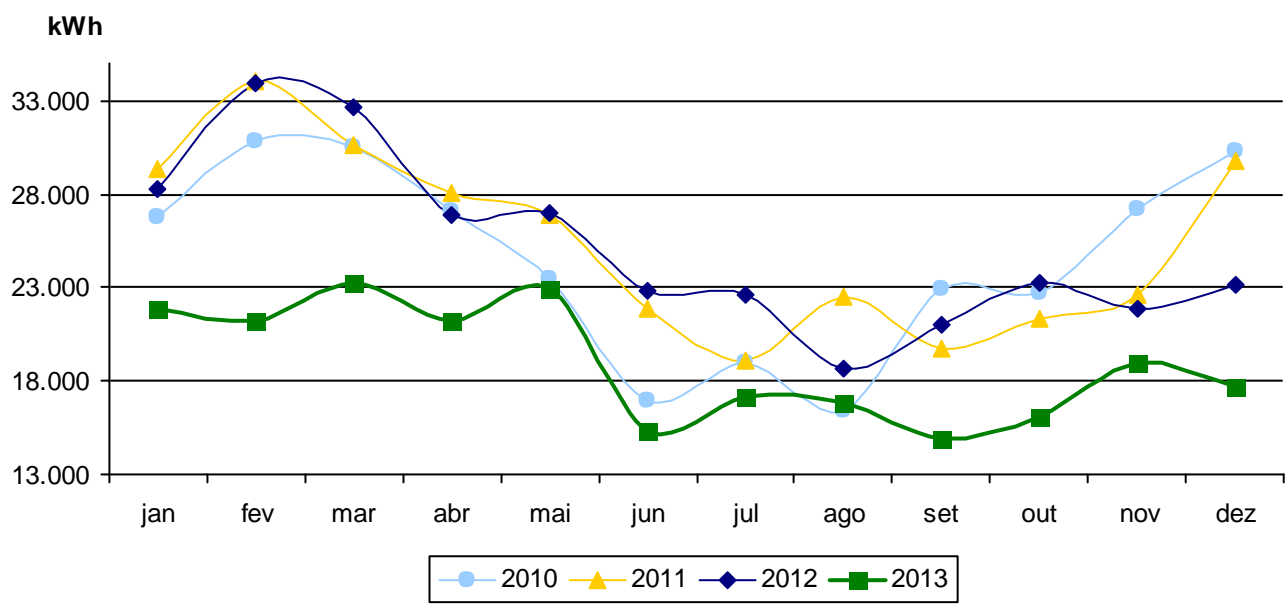

Figura 1. Consumo de energia elétrica da agência nos anos de 2010 a 2013.

Fonte: Faturas da concessionária adaptado pelo autor.

O GHG protocol (2012) sugere que uma média de vários anos pode ajudar a minimizar as flutuações anormais nas emissões de GEE, as quais fariam com que os dados de apenas um ano não sejam representativos do perfil típico de emissões da empresa. Para definição da linha-base de consumo foi excluído o ano de 2012 por apresentar variações no consumo devido ao período de execução dos serviços de instalação dos novos equipamentos. Na Tabela 4 consta o consumo de energia elétrica e a média dos anos anteriores de 2010 a 2011 . A média anual foi de $300.074 \mathrm{kWh}$.

Tabela 4 - Consumo de energia elétrica - linha-base (kWh)

\begin{tabular}{|c|c|c|c|c|c|c|c|c|c|c|c|c|c|}
\hline \multirow{2}{*}{ Ano } & \multicolumn{12}{|c|}{ Mês } & \multirow{2}{*}{ Total } \\
\hline & Jan & Fev & Mar & Abr & Mai & Jun & Jul & Ago & Set & Out & Nov & Dez & \\
\hline 2010 & 26.739 & 30.878 & 30.537 & 27.115 & 23.438 & 16.983 & 19.030 & 16.437 & 22.967 & 22.710 & 27.223 & 30.276 & 294.333 \\
\hline 2011 & 29.312 & 34.047 & 30.617 & 28.106 & 26.870 & 21.817 & 19.054 & 22.548 & 19.753 & 21.300 & 22.602 & 29.788 & 305.814 \\
\hline Média & 28.026 & 32.463 & 30.577 & 27.611 & 25.154 & 19.400 & 19.042 & 19.493 & 21.360 & 22.005 & 24.913 & 30.032 & 300.074 \\
\hline
\end{tabular}


A análise do consumo de energia elétrica do ano de 2013 resultou em uma economia de $72.553 \mathrm{kWh}$, conforme a Tabela 5, em relação à linha-base (2010 a 2011). A redução percentual de consumo de energia elétrica em 2013 foi de $24,18 \%$.

Tabela 5 - Consumo de energia elétrica - projeto retrofit $(\mathrm{kWh})$

\begin{tabular}{|c|c|c|c|c|c|c|c|c|c|c|c|c|c|}
\hline \multirow{2}{*}{ Ano } & \multicolumn{12}{|c|}{ Mês } & \multirow{2}{*}{ Total } \\
\hline & Jan & Fev & Mar & Abr & Mai & Jun & Jul & Ago & Set & Out & Nov & Dez & \\
\hline base & 28.026 & 32.463 & 30.577 & 27.611 & 25.154 & 19.400 & 19.042 & 19.493 & 21.360 & 22.005 & 24.913 & 30.032 & 300.074 \\
\hline 2013 & 21.872 & 21.219 & 23.211 & 21.258 & 22.896 & 15.360 & 17.180 & 16.841 & 14.885 & 16.123 & 18.982 & 17.694 & 227.521 \\
\hline \multicolumn{13}{|c|}{ Diferença $=($ base -2013$)$} & 72.553 \\
\hline \multicolumn{13}{|c|}{ Variação percentual } & $24,18 \%$ \\
\hline
\end{tabular}

Para o cálculo da economia financeira adotou-se o custo unitário ( $\mathrm{R} \$ / \mathrm{kWh}$ ) médio do ano de 2013 de $\mathrm{R} \$ 0,37$ multiplicado pelo consumo de energia (kWh) da linha-base (2010 a 2011). Este seria o custo caso o retrofit não tivesse sido executado. Este método evita que reajustes na tarifa (aumento ou desconto) distorçam o valor do custo. A economia financeira do ano de 2013 resultou uma redução de $\mathrm{R} \$ 26.877,45$, conforme a Tabela 6 , em relação à linha-base.

Tabela 6 - Economia financeira $(\mathrm{R} \$)$

\begin{tabular}{|c|c|c|c|c|c|c|c|c|c|c|c|c|c|}
\hline \multirow{2}{*}{ Ano } & \multicolumn{12}{|c|}{ Mês } & \multirow{2}{*}{ Total } \\
\hline & Jan & Fev & Mar & Abr & Mai & Jun & Jul & Ago & Set & Out & Nov & Dez & \\
\hline base & 10.382 & 12.026 & 11.327 & 10.228 & 9.318 & 7.187 & 7.054 & 7.221 & 7.913 & 8.152 & 9.229 & 11.126 & $111.164,00$ \\
\hline 2013 & 8.668 & 7.657 & 7.712 & 7.220 & 7.847 & 5.392 & 6.155 & 6.579 & 6.211 & 6.721 & 7.212 & 6.911 & $84.286,00$ \\
\hline Difer & $a=(b$ & -2013 & & & & & & & & & & $\mathbf{R} \$$ & $26.877,45$ \\
\hline
\end{tabular}

\subsection{Inventário de emissões de GEE}

O GHG Protocol (2012) define o Potencial de Aquecimento Global (PAG) como o fator que descreve o impacto do forçamento radiativo (grau de dano à atmosfera) de uma unidade de determinado GEE relativamente a uma unidade de $\mathrm{CO}_{2}$. Na Tabela 7 é apresentado qual o gás refrigerante em cada sistema (soma de todos os equipamentos), a carga inicial total de gás ( $\mathrm{kg}$ ) e o PAG. Multiplicando a carga de gás refrigerante pelo respectivo PAG temos o potencial de gás carbônico equivalente $\left(\mathrm{CO}_{2} \mathrm{eq}\right)$.

Tabela 7 - Carga de gás e $\mathrm{CO}_{2}$ eq dos sistemas.

\begin{tabular}{lccccc}
\hline Situação & Gás & PAG $^{1}$ & Carga $^{\mathbf{2}}$ & \multicolumn{2}{c}{$\mathbf{C O}_{\mathbf{2}} \mathbf{e q}$} \\
\hline Anterior & & & $\mathbf{k g}$ & $\mathbf{k g}$ & $\mathbf{t}$ \\
\hline Retrofit & HCFC-22 & 1.760 & 92,5 & $162.800,0$ & 162,80 \\
\hline Variação percentual & 2.088 & 60,5 & $126.324,0$ & 126,32 \\
\hline
\end{tabular}

Fonte: ${ }^{1}$ IPCC, 2013; ${ }^{2}$ Placa de identificação dos equipamentos.

Apesar do gás do retrofit, R-410A, ter um PAG 18,6\% superior ao HCFC-22, do sistema anterior, a redução da carga total de gás no sistema de $34,6 \%$ resulta em uma redução de $22,4 \%$ na possibilidade de emissões. Esta diferença é devida ao risco de vazamento do gás para a atmosfera em caso de falha nos equipamentos.

O Guia para Inventários Nacionais de Gases de Efeito Estufa do IPCC (2006) recomenda que a taxa de vazamento anual para equipamentos comerciais seja: $10 \%$ da carga inicial para equipamentos novos e até $35 \%$ para equipamentos no final da vida útil. O vazamento do sistema anterior foi de 56,98 toneladas de $\mathrm{CO}_{2}$ eq e do retrofit de 12,63 toneladas de $\mathrm{CO}_{2} \mathrm{eq}$. O resultado foi 
uma mitigação anual de 44,35 toneladas de $\mathrm{CO}_{2}$ eq. $\mathrm{O}$ percentual de redução entre a situação atual e o retrofit foi de $77,83 \%$.

Para o cálculo da mitigação adotaram-se os fatores de emissão do ano de 2013, pois esta seria a emissão caso o retrofit não tivesse sido executado. Com a redução no consumo de energia elétrica foram mitigadas (não emitidas) 6,96 toneladas de $\mathrm{CO}_{2}$ no ano do inventário, conforme a Tabela 8.

Tabela 8 - Mitigação da emissão $\left(\mathrm{tCO}_{2}\right)$

\begin{tabular}{|c|c|c|c|c|c|c|c|c|c|c|c|c|c|}
\hline \multirow{2}{*}{ Ano } & \multicolumn{12}{|c|}{ Mês } & \multirow{2}{*}{ Total } \\
\hline & Jan & Fev & Mar & Abr & Mai & Jun & Jul & Ago & Set & Out & Nov & Dez & \\
\hline base & 3,2257 & 3,5384 & 2,9996 & 2,6478 & 2,8952 & 2,0933 & 1,5957 & 1,6237 & 1,7942 & 1,8286 & 2,3169 & 2,5257 & 29,0849 \\
\hline 2013 & 2,5175 & 2,3129 & 2,2770 & 2,0386 & 2,6353 & 1,6573 & 1,4397 & 1,4029 & 1,2503 & 1,3398 & 1,7653 & 1,4881 & 22,1247 \\
\hline \multicolumn{13}{|c|}{ Diferença $=($ base -2013$)$} & 6,9602 \\
\hline
\end{tabular}

Os equipamentos existentes (no final da vida útil) foram destinados para reciclagem das peças de aço, cobre e polímeros diversos. Todo o gás existente $(92,5 \mathrm{~kg}$ de HCFC-22) foi recolhido e destinado para reutilização na manutenção de outros equipamentos. Desta forma, não ocorreram emissões de GEE devido ao descarte dos equipamentos.

Na Tabela 9 está demonstrada a mitigação anual direta pela substituição do gás refrigerante (escopo 1), de 44,35 toneladas de $\mathrm{CO}_{2}$ eq, a mitigação anual indireta pela redução no consumo de energia elétrica (escopo 2), de 6,96 toneladas de $\mathrm{CO}_{2}$, e a emissão específica devido ao descarte dos equipamentos (escopo 3), que foi nula. A soma dos escopos resultou em uma mitigação total anual de 51,31 toneladas de $\mathrm{CO}_{2}$ eq.

Tabela 9 - Mitigação total anual $\left(\mathrm{tCO}_{2} \mathrm{eq}\right)$

\begin{tabular}{ccccc}
\hline Ano & Escopo 1 & Escopo 2 & Escopo 3 & Total \\
\hline base & 56,98 & 29,08 & - & $\mathbf{8 6 , 0 6}$ \\
\hline 2013 & 12,63 & 22,12 & - & $\mathbf{3 4 , 7 6}$ \\
\hline (base - 2013) & 44,35 & 6,96 & - & $\mathbf{5 1 , 3 1}$ \\
\hline
\end{tabular}

\subsection{Indicadores ambientais}

$\mathrm{O}$ indicador de emissão de carbono na linha-base (sistema anterior) foi de 54,03 $\mathrm{kgCO}_{2} \mathrm{eq} / \mathrm{m}^{2} /$ ano. No ano do inventário (retrofit) foi de $21,82 \mathrm{kgCO}_{2} \mathrm{eq} / \mathrm{m}^{2} / \mathrm{ano}$. O resultado é uma redução de 59,62\%. Não é possível comparar diretamente este fator com os da ferramenta CarbonBuzz devido as diferenças do clima e das fontes de geração de energia.

Deixaram de ser emitidas (mitigadas) para a atmosfera $51,31 \mathrm{tCO}_{2}$ eq no ano do inventário. Considerando o fator de fixação de 10,45 árvores/ $/ \mathrm{CO}_{2}$ eq a mitigação anual de GEE é equivalente à quantidade de $\mathrm{CO}_{2}$ absorvida da atmosfera de um reflorestamento com 536 árvores. O indicador de consumo de energia na linha-base (sistema anterior) foi de $188,37 \mathrm{kWh} / \mathrm{m}^{2} / \mathrm{ano}$. No ano do inventário (retrofit) foi de $142,83 \mathrm{kWh} / \mathrm{m}^{2} /$ ano. O resultado é uma redução de $24,18 \%$.

Comparando os resultados com a pesquisa de Borgstein e Lamberts (2014) em que a média de consumo das agências bancárias da cidade de Florianópolis é de 193,25 kWh/m²/ano, conclui-se que a situação anterior apresentava um consumo de energia elétrica próximo ao valor típico e que a situação após o retrofit apresenta um consumo mais eficiente que as demais agências bancárias. 


\subsection{Indicadores financeiros}

O fluxo de caixa, conforme a Tabela 10, apresenta os valores de investimento inicial de $\mathrm{R} \$$ 399.269,57 (incluídos os equipamentos, mão-de-obra, transportes, lucro e impostos) e as receitas anuais devido à economia de energia de $\mathrm{R} \$ 26.887,45$.

Adotou-se um horizonte de planejamento de 15 anos, que é expectativa de vida útil média do equipamento (IPCC, 2006), e taxa mínima de atratividade (TMA) de 7,25\% ao ano, conforme taxa SELIC (BCB, 2014) de novembro de 2012 vigente na data de conclusão e pagamento da obra.

Tabela 10 - Fluxo de caixa e indicadores financeiros

\begin{tabular}{|c|c|c|c|c|}
\hline Ano & & eto & VP Negativo & VP Positivo \\
\hline & 0 & -R\$ 399.269,57 & -R\$ 399.269,57 & \\
\hline & 1 & $\mathrm{R} \$ 26.887,45$ & & $\mathrm{R} \$ 25.069,88$ \\
\hline & 2 & $\mathrm{R} \$ 26.887,45$ & & $\mathrm{R} \$ 23.375,18$ \\
\hline & 3 & $\mathrm{R} \$ 26.887,45$ & & $\mathrm{R} \$ 21.795,04$ \\
\hline & 4 & $\mathrm{R} \$ 26.887,45$ & + & $\mathrm{R} \$ 20.321,72$ \\
\hline & 5 & $\mathrm{R} \$ 26.887,45$ & & $\mathrm{R} \$ 18.947,99$ \\
\hline & 6 & $\mathrm{R} \$ 26.887,45$ & & $\mathrm{R} \$ 17.667,12$ \\
\hline & 7 & $\mathrm{R} \$ 26.887,45$ & & $\mathrm{R} \$ 16.472,84$ \\
\hline & 8 & $\mathrm{R} \$ 26.887,45$ & & $\mathrm{R} \$ 15.359,29$ \\
\hline & 9 & $\mathrm{R} \$ 26.887,45$ & & $\mathrm{R} \$ 14.321,02$ \\
\hline & 10 & $\mathrm{R} \$ 26.887,45$ & & $\mathrm{R} \$ 13.352,93$ \\
\hline & 11 & $\mathrm{R} \$ 26.887,45$ & & $\mathrm{R} \$ 12.450,29$ \\
\hline & 12 & $\mathrm{R} \$ 26.887,45$ & & $\mathrm{R} \$ 11.608,66$ \\
\hline & 13 & $\mathrm{R} \$ 26.887,45$ & & $\mathrm{R} \$ 10.823,92$ \\
\hline & 14 & $\mathrm{R} \$ 26.887,45$ & & $\mathrm{R} \$ 10.092,24$ \\
\hline & 15 & $\mathrm{R} \$ 26.887,45$ & . & $\mathrm{R} \$ 9.410,01$ \\
\hline TOTAL & & & -R\$ 399.269,57 & R\$241.068,13 \\
\hline TMA & & $7,25 \%$ & Taxa mínima de atrati & vidade \\
\hline VPL & & -R\$ 158.201,44 & Valor Presente Líqui & ido \\
\hline IBC & & $\mathbf{0 , 6 0}$ & Índice Benefício/Cus & \\
\hline Payback & & 14,85 & Anos & - \\
\hline
\end{tabular}

O Índice Benefício/Custo (IBC) do projeto resultou o valor 0,60. Pode-se concluir que haverá retorno de $60 \%$ do investimento inicial pela redução do consumo de energia elétrica ao longo do tempo. O tempo de retorno do investimento (payback) ocorre em 14,85 anos, dentro da estimativa de vida útil média do equipamento que é de 15 anos. Analisou-se duas alternativas de investimento:

Anterior: Caso fosse mantido o sistema anterior, devido ao consumo de energia ao longo de 15 anos, o custo total seria um valor presente (VP) de R\$241.068,13.

Retrofit: O sistema do retrofit tem investimento inicial de $\mathrm{R} \$ 399.269,57$. Descontado a economia de energia de $\mathrm{R} \$ 241.068,13$ é igual a um valor presente líquido (VPL) de R\$ 158.201,44.

Comparando as duas alternativas o projeto de retrofit é mais vantajoso em relação à alternativa de manter o sistema existente. 


\section{CONCLUSÕES}

Um ano após a execução da obra de retrofit foi atingido o objetivo esperado, que era substituir o sistema de ar condicionado antigo por um novo com mais eficiência energética, com redução do consumo de energia elétrica e a consequente redução do impacto ambiental.

A redução do consumo de energia elétrica foi de $24,18 \%$. Comparando os resultados com a média de consumo das agências bancárias da cidade de Florianópolis, conclui-se que a situação anterior apresentava um consumo de energia elétrica de $188,37 \mathrm{kWh} / \mathrm{m}^{2} /$ ano próximo ao valor típico e que a situação após o retrofit apresenta um consumo eficiente de $142,83 \mathrm{kWh} / \mathrm{m}^{2} / \mathrm{ano}$.

A economia financeira no ano 2013 foi de $\mathrm{R} \$ 26.887,45$. No horizonte de planejamento de 15 anos a economia projetada é de $\mathrm{R} \$ 241.068,13$. Com o índice benefício/custo (IBC) de 0,6 conclui-se que haverá retorno de $60 \%$ do investimento inicial pela redução do consumo de energia elétrica ao longo do tempo. O retorno do investimento (payback) ocorre em 14,85 anos, dentro da estimativa de vida útil média dos equipamentos.

A mitigação dos gases de efeito estufa (GEE) foi de 51,31 toneladas de $\mathrm{CO}_{2}$ eq no ano do inventário, devido ao aumento da eficiência energética dos novos equipamentos, a substituição do tipo de gás e a redução da carga de gás refrigerante. O impacto ambiental anual é equivalente ao plantio de 536 árvores. Durante a vida útil do sistema de 15 anos o impacto projetado é de 8.041 árvores.

Pode-se concluir que o projeto de retrofit é sustentável quanto aos aspectos ambientais e financeiros, conforme os indicadores analisados neste estudo. Considerando o caso em estudo é recomendável aos gestores de edificações comerciais executarem o retrofit do sistema de ar condicionado.

Para futuras pesquisas sugere-se: Pesquisas sobre a quantidade de vazamento de gás durante a operação dos equipamentos possibilitariam um cálculo preciso das emissões devido às falhas nos equipamentos. E que contratos de manutenção incluam relatórios anotando a carga de gás de reposição devido a vazamentos para a atmosfera.

\section{AGRADECIMENTOS}

À instituição bancária pelo incentivo e apoio financeiro.

\section{REFERÊNCIAS}

ASSOCIAÇÃO BRASILEIRA DE NORMAS TÉCNICAS (ABNT). NBR 16.401-1. Instalações de ar-condicionado - Sistemas centrais e unitários Parte 1: Projetos das instalações, Rio de Janeiro, 2008.

BANCO CENTRAL DO BRASIL (BCB). Taxa Selic. Disponível em: <http://www.bcb.gov.br/?SELICDIARIOS>. Acesso em: 09 set. 2014.

BORGSTEIN, E.; LAMBERTS, R. Developing energy consumption benchmarks for buildings: bank branches in Brazil. Energy and Buildings, 2014. Disponível em: <http://dx.doi.org/10.1016/j.enbuild.2014.07.028>. Acesso em: 09 set. 2014. 
BRASIL. Lei no 12.187, de 29 de dezembro de 2009. Disponível em:

<http://www.planalto.gov.br/ccivil_03/_ato2007-2010/2009/lei/112187.htm>. Acesso em: 09 set. 2014.

. Decreto $\mathbf{n}^{\mathbf{0}}$ 7.390, de 9 de dezembro de 2010. Disponível em:

<http://www.planalto.gov.br/ccivil_03/_Ato2007-2010/2010/Decreto/D7390.htm>. Acesso em: 09 set. 2014.

CAMARGO, C. Análise de Investimentos e Demonstrativos Financeiros. 1. ed. Curitiba: IBPEX, 2007. p.79-116.

CARBONBUZZ. Benchmark Sector: Offices. 2014. Disponível em:

<http://www.carbonbuzz.org/sectorbreakdown.jsp?id=2>. Acesso em: 09 set. 2014.

CONSELHO BRASILEIRO DE CONSTRUÇÕES SUSTENTÁVEIS (CBCS). Plataforma de Cálculo Benchmarking. 2014. Disponível em: < http://www.cbcs.org.br/website/benchmarkingplataforma/>. Acesso em: 09 set. 2014.

FLIZIKOWSKI, L.C. Estimativa de emissões de dióxido de carbono na construção civil e neutralização com espécies florestais: um estudo de caso. $121 \mathrm{f}$. Dissertação (Mestrado em Engenharia Florestal) - Universidade Federal do Paraná, Curitiba, 2012.

GHG PROTOCOL BRASIL (GHG). Contabilização, quantificação e publicação de inventários corporativos de emissões de Gases de Efeito Estufa. 2. ed. São Paulo, 2012. Disponível em: <http://ghgprotocolbrasil.com.br/arquivos/152/especificacoes_pb_ghgprotocol.pdf >. Acesso em: 09 set. 2014.

INTERGOVERNMENTAL PANEL ON CLIMATE CHANGE (IPCC). Climate Change 2013: Anthropogenic and Natural Radiative Forcing. 2013. Disponível em:

<http://www.climatechange2013.org/images/report/WG1AR5_Chapter08_FINAL.pdf>. Acesso em: 09 set. 2014.

Impacts, Adaptation, and Vulnerability. 2014a. Disponível em: <http://ipcc-

Climate Change 2014:

wg2.gov/AR5/images/uploads/WG2AR5_SPM_FINAL.pdf>. Acesso em: 09 set. 2014.

Mitigation of Climate Change. 2014b. Disponível em

Climate Change 2014:

<http://report.mitigation2014.org/spm/ipcc_wg3_ar5_summary-for-policymakers_approved.pdf >. Acesso em: 09 set. 2014.

Guidelines for National

Greenhouse Gas Inventories, Greenhouse Gas Inventory: Industrial Processes and Product Use. 2006. Disponível em: <http://www.ipcc-nggip.iges.or.jp/public/2006gl/vol3.html>. Acesso em: 09 set. 2014.

MINISTÉRIO DA CIÊNCIA E TECNOLOGIA (MCT). Fatores de emissão de $\mathbf{C O}_{2}$ pela energia elétrica no Sistema Interligado Nacional do Brasil. 2013. Disponível em:

<http://www.mct.gov.br/index.php/content/view/321144.html\#ancora>. Acesso em: 09 set. 2014. 
MINISTÉRIO DO MEIO AMBIENTE (MMA). Programa Brasileiro de eliminação dos HCFCs, 2012. Disponível em:

<http://www.mma.gov.br/estruturas/ozonio/_arquivos/3_anexo_ii__pbh_final_17_02_2012_130.p df>. Acesso em: 09 set. 2014.

SANQUETTA, C.R.; FLIZIKOWSKI, L.C.; DALLA CORTE, A.P.; MOGNON, F.; MAAS, G.C.B. Estimativa das emissões de gases de efeito estufa em uma obra de construção civil com a metodologia GHG protocol. Enciclopédia Biosfera, Centro Científico Conhecer, Goiânia, v.9, n.16, p. 1094. 2013. 